Original Article

\title{
SPECTRAL ANALYSIS OF RESONANT SOUNDS OF CHEST PERCUSSION
}

\author{
E. Ashwini Kumar ${ }^{1}$, Bharat Vyas Marla ${ }^{1}$, C. Jairaj Kumarr ${ }^{1}$ Garvit Chitkara' ${ }^{1}$ \\ Nilesh Mishra ${ }^{1} \&$ Arunachalam Kumar ${ }^{2}$ \\ Correspondence : \\ Arunachalam Kumar \\ Director (R \& D) Nitte University, Mangalore 575018, India \\ E-mail : DirectorRD@ nitte edu.in
}

${ }^{1}$ Formerly Interns, Kasturba Medical College, M anipal University, M angalore - 575 001, India. ${ }^{2}$ Professor of Anatomy, K.S. Hegde M edical Academy, Nitte University, M angalore - 575 018, India.

\begin{abstract}
:
General clinical examination, in the consulting room or in the out-patient department determines not only a provisional diagnosis but also provides the clinician information whether the patient needs hospitalisation and further investigation. Inspection, auscultation, palpation and percussion forms the basis of clinical examination.

Whilst much has been described and discussed on the first three procedures, inspection, auscultation and palpation, far less attention has been focused on the correct method and diagnostic significance of percussion.

In this brief communication we discuss the results obtained through a computerized spectral analysis of percussion sounds. It is suggested that, noting the high accuracy and reliability of spectral percussion sound read-out analysis, medical equipment designers manufacturers could well bring out a simple hand-held device which could analyse the percussion sounds to yield the most prominent of amplitude and frequency; such ready and reliable information that could be of much diagnostic and prognostic significance.
\end{abstract}

Keywords : chest percussion, spectral analysis, amplitude, frequency

\section{Introduction :}

Percussion is a well-known clinical method to diagnose the underlying pathology at various sites, using the principles of resonance of sound by a hallow cavity. However no effort has been made to quantify these resonance characteristics which may enhance the tool. This paper throws light on the possibility of this idea and the ways and means to make it happen ${ }^{1}$.

\section{M aterials and Methods :}

Standard percussion procedure detailed in the basic clinical method texts is followed. Healthy individuals of same age group are selected randomly. A standard microphone to

\begin{tabular}{l} 
Access this article online \\
\hline Quick Response Code
\end{tabular}

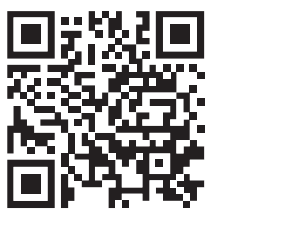
record the sounds of percussion is placed on the sternum at the angle of Louis. For simplicity sake only the right side of the chest starting from the $1^{\text {st }}$ intercostal space to $5^{\text {th }}$ intercostal space are percussed through standard methods. The amount of force exerted for each attempt was kept fairly constant. Each intercostal space was percussed ten times to dilute the experimental errors.

The sounds were directly recorded on to the hard disk of a computer using the standard methods of sounds capturing. The gain was kept constant for all the subjects. Sounds thus captures were analyzed with Sony ${ }^{\circledR}$ Sound Forge ${ }^{\circledR V e r s i o n ~} 7.0$ software using spectral analysis to yield most prominent amplitude, frequency and note for each resonant sound. To standardize the accuracy of percussion technique, the experiment is repeated for five attempts percussing each inter costal space for two times from top to bottom. Results thus obtained were tabulated and conclusions were drawn.

\section{Results:}

The average amplitude and frequency of resonance for each intercostal space of each subject are shown in Graphs I\& I. 


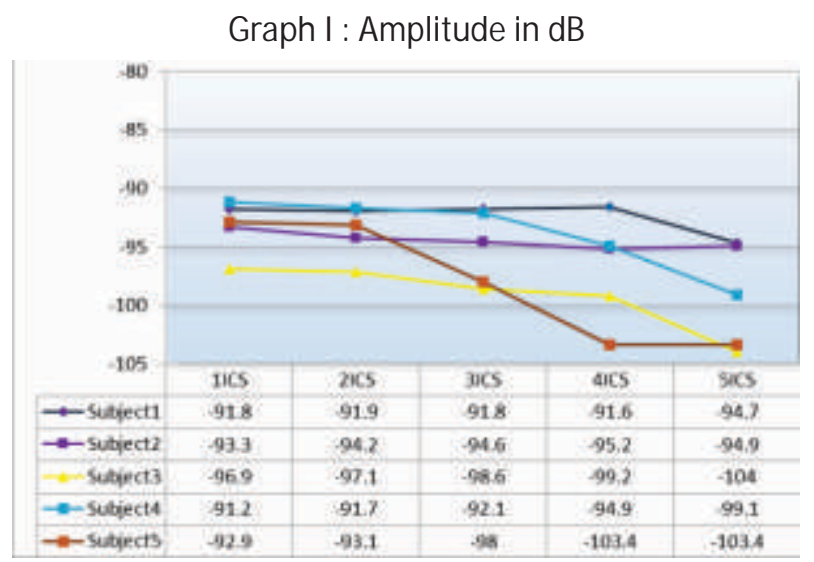

\section{Discussion :}

Average amplitude distribution and frequency distribution reflects the dullness encountered in the $5^{\text {th }}$ intercostal due to the presence of liver. It was also observed that the amplitude of resonance progressively from $1^{\text {st }}$ intercostal space to $4^{\text {th }}$ and then it fell steeply over the $5^{\text {th }}$ intercostal space. Although the individual values varied for each subject, the pattern of rise and fall is common in all healthy individuals.

Any deviation from this would signify the underlying pathology and restoration to this pattern would tell about the remission. If the patterns of deviation are studied in each disease, it should be possible to use to use the amplitude variation and frequency variation as a diagnostic tool.

A simple hand held device which can analyse the sounds

\section{Reference:}

1. Kumar AE, Vyas BV, Kumar JC, Chitkara G, Mishra N \& Arunachalam Kumar, 2005, Spectral analysis of resonant sounds of chest percussion as a non-invasive diagnostic- prognostic tool; Proceedings of the National Conference on Devices, Intelligent Systems \& Communication, Manipal

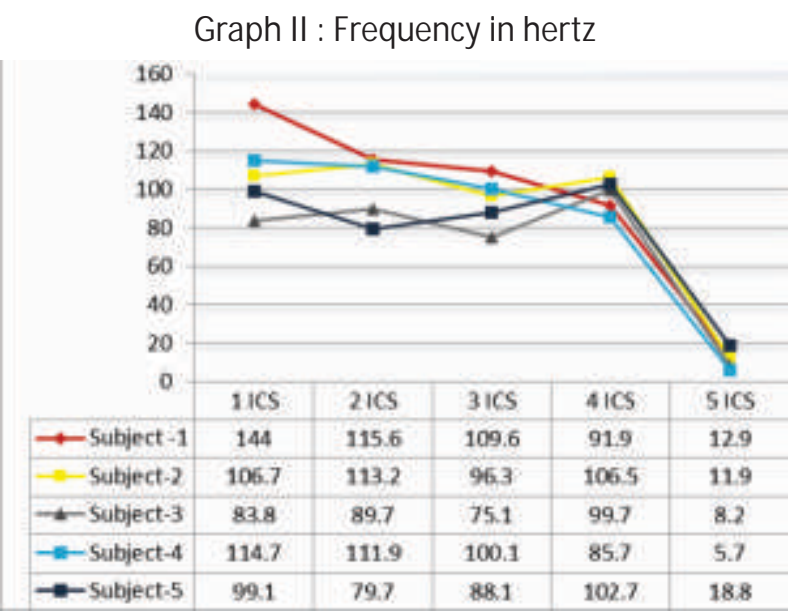

and yield the most prominent amplitude and frequency will be of great importance to physicians in diagnosing the disease conditions where dullness is abnormally present in $1^{\text {st }}$ to $4^{\text {th }}$ intercostal space and also in conditions where there is a pathological increase in resonance in these areas.

It is our inference that a handy portable computerized electronic device designed to evaluate frequency and amplitude of resonant percussion sounds accurately and reliably, will negate any subjective physician bias in interpreting the results of standard percussion sounds, which presently at best, is totally based on aural acuity of the examiner/s.

\section{Acknowledgement:}

This article is a part of a paper presented at a biomedical devices conference in M anipal 